\title{
AVALIAÇÃO DA ESTABILIDADE DE TENSÃO DO SISTEMA BRASILEIRO - UM CASO DE ESTUDO
}

\author{
Carolina de M. Affonso* \\ carolinalufpa.br
}

\author{
Luciano J. da Silva ${ }^{\dagger}$ \\ luciano@furnas.com.br
}

\author{
Luis C. P. da Silva ${ }^{\dagger}$ \\ luiddsee.fee.unicamp.br
}

*Departamento de Engenharia Elétrica e Computação, UFPA, C.P. 8619, CEP 66075-110, Belém, PA, Brasil.

${ }^{\dagger}$ Faculdade de Engenharia Elétrica e de Computação, UNICAMP, C.P. 6101, CEP 13083-970 - Campinas, SP, Brasil.

\begin{abstract}
This paper presents results of a study of the Brazilian system operation in respect to voltage stability criteria. The analyses are applied using real-life data from the operation and planning. The system is divided in 4 regions: North, Northeast, South and Southeast/West Center. System stability margin is evaluated for each one of these areas. The results show that Brazilian system has stability margin much lower than the existing voltage stability criteria, limited by the Southeast/West Center region. A detailed analysis of this critical area is performed. This paper also presents the stability margin behavior during a typical weekday curve and a contingency analysis. The contingency severity is verified by using the modal analysis technique assessing each contingency impact classifying as local, area or systemic.
\end{abstract}

KEYWORDS: Voltage Stability, PV curves, Modal Analysis, Brazilian system.

\section{RESUMO}

Este artigo apresenta resultados de um estudo da atual condi-

Artigo submetido em 17/05/2007

1a. Revisão em 24/01/2008

2a. Revisão em 15/04/2008

Aceito sob recomendação do Editor Associado

Prof. Denis Vinicius Coury ção de operação do sistema interligado brasileiro com relação à estabilidade de tensão. As análises são realizadas a partir de dados reais do planejamento e da operação. O sistema é dividido em 4 regiões: Norte, Nordeste, Sul e Sudeste/CentroOeste. A margem de estabilidade é obtida para cada uma destas regiões. Os resultados mostram que o sistema interligado brasileiro apresenta margem de estabilidade muito abaixo da sugerida pelos critérios existentes, a qual é limitada pela região Sudeste/Centro-Oeste. Uma análise detalhada para esta área crítica do sistema é realizada. Além de uma avaliação do comportamento da margem de estabilidade de tensão durante um dia típico de semana, esta trabalho apresenta uma análise de contingências. Utilizando a metodologia de análise modal verifica-se a abrangência das contingências, mensurando o impacto de cada contingência como local, de área ou sistêmico.

PALAVRAS-CHAVE: Estabilidade de Tensão, Curvas PV, Análise Modal, Sistema Brasileiro.

\section{INTRODUÇÃO}

O constante crescimento da demanda de energia, investimentos insuficientes no sistema e a reestruturação do setor elétrico tem contribuído para a operação dos sistemas de energia elétrica nas proximidades de seu limite de transmissão, comprometendo o atendimento de alguns critérios de segurança 
(Kabouris et al., 2000). Nesse novo contexto, tem-se tornado cada vez mais importante determinar quão distante o sistema encontra-se operando de seu limite de estabilidade de tensão.

A instabilidade de tensão ocorre quando uma perturbação causa uma queda progressiva e irreversível na tensão em uma ou mais barras da rede, estando associada principalmente à falta de suporte de potência reativa em situações extremas de carregamento (Kundur, 1994; IEEE/CIGRE, 2004). Diversos países já relataram casos de instabilidade de tensão com grandes prejuízos financeiros, como o grande blecaute ocorrido recentemente em agosto de 2003 afetando parte do Canadá e Estados Unidos (U.S.- Canada Task Force, 2004; Corsi and Sabelli, 2004).

Por esta razão, diversos agentes reguladores da América do Norte têm implementado critérios de segurança nas normas de acesso a transmissão, como o WECC (Western Electricity Coordinating Council) que sugere o atendimento de uma margem mínima de 5\% considerando contingências simples $(\mathrm{N}-1), 2,5 \%$ para contingências duplas e margem maior do que zero para o caso de múltiplas contingências (perda simultânea da combinação de 3 ou mais elementos do sistema). Além disso, recomenda-se que a margem de estabilidade para o sistema em condições normais de operação seja maior do que a margem para o caso N-1 (WECC, 1998).

O Operador Nacional do Sistema Elétrico (ONS) do Brasil também iniciou estudos recomendando o atendimento de uma margem mínima de $6 \%$ para o caso de contingências simples (ONS, 2002). No entanto, na prática, requerimentos de estabilidade de tensão ainda não são considerados na operação e planejamento do sistema brasileiro. A importância de avaliar a margem de estabilidade de tensão do sistema é tão grande que recentemente diversas pesquisas tem sido feitas para a estimação e monitoramento da margem de estabilidade de tensão do sistema em tempo real (Amjady, 2004; Bao et al.,2003; Kamalasadan et al., 2006).

Este artigo tem como principal contribuição avaliar a atual condição de operação do sistema interligado brasileiro utilizando dados reais do planejamento da operação, identificando suas áreas críticas. Através da análise modal, podese verificar quais contingências são verdadeiramente severas, identificando a área geográfica atingida por um provável colapso de tensão. Deve-se ressaltar a importância desta análise, uma vez que estudos deste nível ainda não foram desenvolvidos para o sistema brasileiro até o momento.

Este artigo está organizado como segue. A metodologia utilizada para avaliação da margem de estabilidade de tensão e análise modal é discutida na seção 2. A seção 3 apresenta a avaliação da margem de estabilidade do sistema interligado brasileiro. A seção 4 apresenta a avaliação da margem de estabilidade e análise de contingências para a área crítica do sistema, que é a região de São Paulo. A seção 5 apresenta as principais conclusões obtidas com esta pesquisa.

\section{METODOLOGIA}

A análise da estabilidade de tensão envolve o conhecimento de dois aspectos importantes:

- Identificar o quão próximo o sistema está operando do ponto de colapso de tensão;

- Identificar quais áreas são mais sensíveis a estabilidade de tensão, de modo a se estudar possíveis modificações no sistema para ação de medidas corretivas.

\subsection{Curva PV}

Os métodos baseados nas curvas PV informam a margem de estabilidade de tensão do sistema (MET), que é a medida da distância, em MW ou em percentual, do ponto base de operação até o ponto de máximo carregamento do sistema (Taylor, 1994). Para cada aumento no carregamento do sistema um problema de fluxo de carga é resolvido, e os pontos de equilíbrio obtidos definem a curva PV. Os métodos estáticos, principalmente os baseados no levantamento de curvas PV, permitem a avaliação rápida e simples das condições críticas do sistema elétrico, podendo-se analisar diversos cenários.

A avaliação da margem de estabilidade de tensão pode ser obtida de dois modos: para todo o sistema e para áreas selecionadas do sistema. No primeiro caso, a margem de estabilidade é obtida para todo o sistema aumentando-se o carregamento de todas as barras de carga da rede de modo proporcional ao carregamento do caso base (mantendo o fator de potência constante). Toda a geração do sistema é ajustada de modo a atender a nova demanda do sistema durante o processo de construção da curva PV. No segundo caso, diferentes margens de estabilidade são obtidas para cada área do sistema. Para cada área de estudo, aumenta-se o carregamento da área mantendo fator de potência constante. A geração externa a área de estudo permanece constante, sendo o excedente de carga suprido apenas pelos geradores internos da área de estudo. Este procedimento conservador permite identificar quais áreas são mais vulneráveis a um colapso de tensão [Força Tarefa, 1999].

Neste artigo, a margem de estabilidade foi avaliada utilizando o método da continuação para todo o sistema e para áreas selecionadas do sistema. Em todos os casos a modelagem de carga utilizada foi de potência constante (situação mais pessimista) seguindo as recomendações do WECC (WECC, 1998). Assim, o ponto de máximo carregamento coincide com o ponto limite de estabilidade de tensão. Também foram considerados os limites de potência reativa dos 
geradores no processo de construção da curva PV para obtenção de resultados mais realistas.

\subsection{Análise Modal}

Apesar de fornecer informação importante com relação a margem de estabilidade de tensão do sistema, o método de curvas PV não propicia a identificação de barras ou áreas críticas do ponto de vista da estabilidade de tensão, as quais representam os locais mais deficientes do sistema, e conseqüentemente, as melhores opções para a aplicação de medidas de reforço contra o problema de instabilidade. Através da análise modal pode-se identificar as áreas críticas do sistema e também verificar a abrangência das contingências.

As equações do modelo estático do sistema de potência linearizado em torno de um ponto de equilíbrio podem ser escritas na forma:

$$
\left[\begin{array}{c}
\Delta P \\
\Delta Q
\end{array}\right]=\left[\begin{array}{ll}
J_{P \theta} & J_{P V} \\
J_{Q \theta} & J_{Q V}
\end{array}\right]\left[\begin{array}{c}
\Delta \theta \\
\Delta V
\end{array}\right]=J\left[\begin{array}{c}
\Delta \theta \\
\Delta V
\end{array}\right]
$$

onde $\Delta \mathrm{P}$ são variações de potência ativa, $\Delta \mathrm{Q}$ são variações de potência reativa, $\Delta \theta$ são variações de ângulo, $\Delta \mathrm{V}$ são variações no módulo da tensão e J é a matriz Jacobiana do sistema.

Duas matrizes Jacobianas reduzidas $\left(\mathrm{J}_{R Q V}, \mathbf{J}_{R P \theta}\right)$ podem ser definidas:

$$
\begin{gathered}
\Delta \theta=J_{R P \theta}^{-1} \Delta P, \text { fazendo } \Delta Q=0 \\
\Delta V=J_{R Q V}^{-1} \Delta Q, \text { fazendo } \Delta P=0 \\
J_{R P \theta}=J_{P \theta}-J_{P V} J_{Q V}^{-1} J_{Q \theta} \\
J_{R Q V}=J_{Q V}-J_{Q \theta} J_{P \theta}^{-1} J_{P V}
\end{gathered}
$$

A análise modal estática convencional avalia os autovalores e autovetores críticos da matriz Jacobiana reduzida $\mathbf{J}_{R Q V}$ no ponto de singularidade, como mostra a equação 6 , onde $\Phi$ é a matriz dos autovetores à direita de $\mathrm{J}_{R Q V}, \Gamma$ é a matriz dos autovetores à esquerda de $\mathrm{J}_{R Q V}$ e $\Lambda$ é a matriz diagonal dos autovalores de $\mathbf{J}_{R Q V}$. A partir daí, o fator de participação reativo (FPR) é definido como sendo o produto dos autovetores esquerdo e direito de $\mathbf{J}_{R Q V}$. Estes fatores são amplamente utilizados para indicar as áreas mais adequadas para compensação de potência reativa, uma vez que associam a potência reativa com variações de tensão (Kundur, 1994).

$$
J_{R Q V}=\Phi \Lambda \Gamma
$$

De modo similar, o fator de participação ativo (FPA) pode ser definido como sendo o produto dos autovetores esquerdo e direito de $\mathrm{J}_{R P \theta}$ (Da Silva et al., 2002). Os FPA indicam as áreas mais vulneráveis (de impacto negativo) relacionadas com pro-blemas de instabilidade de tensão a partir de uma perspectiva de variações de potência ativa e estão associados com cada gerador e barra de carga da rede. Para barras de carga, estes fatores indicam as barras mais adequadas a desligamentos emergenciais de carga.

A vantagem da análise modal é que, ao contrário de muitos índices tais como o mínimo valor singular, os autovetores críticos não apresentam variações tão bruscas nas proximidades do ponto de colapso de tensão. Isto significa que as áreas críticas do sistema não mudam abruptamente devido a pequenas variações no carregamento e configuração do sistema. Além disso, a direção ótima indicada pelos fatores de participação garante que a injeção de potência reativa e o corte de carga seja o mínimo necessário para aumentar a margem de estabilidade do sistema (Affonso et al., 2004).

\section{AVALIAÇÃO DA ESTABILIDADE DE TENSÃO DO SIN}

Nesta seção são apresentados os resultados das análises realizadas para o Sistema Interligado Nacional (SIN) represetnativo do sistema brasileiro.

\subsection{Sistema interligado nacional brasi- leiro}

Para estas análises foram utilizados dados reais do sistema interligado brasileiro referente ao planejamento elétrico mensal de fevereiro de 2006, os quais estão disponíveis no site do ONS (www.ons.org.br). Este sistema é composto por 3.451 barras, 309 geradores , 4.914 ramos, 11 compensadores estáticos de reativos e 4 compensadores série-controlável. A rede básica de transmissão compreende as tensões de $230 \mathrm{kV}$ a $750 \mathrm{kV}$. O ponto de operação analisado refere-se ao caso de carga pesada.

O sistema está dividido em 73 áreas representando concessionárias, centros de geração, centros de carga, grandes troncos de transmissão e outros. Assim, algumas áreas não possuem geração alguma para suprir um incremento de carga ocorrido nesta área, e outras áreas não possuem carga alguma para ser incrementada. Deste modo, foram consideradas como 


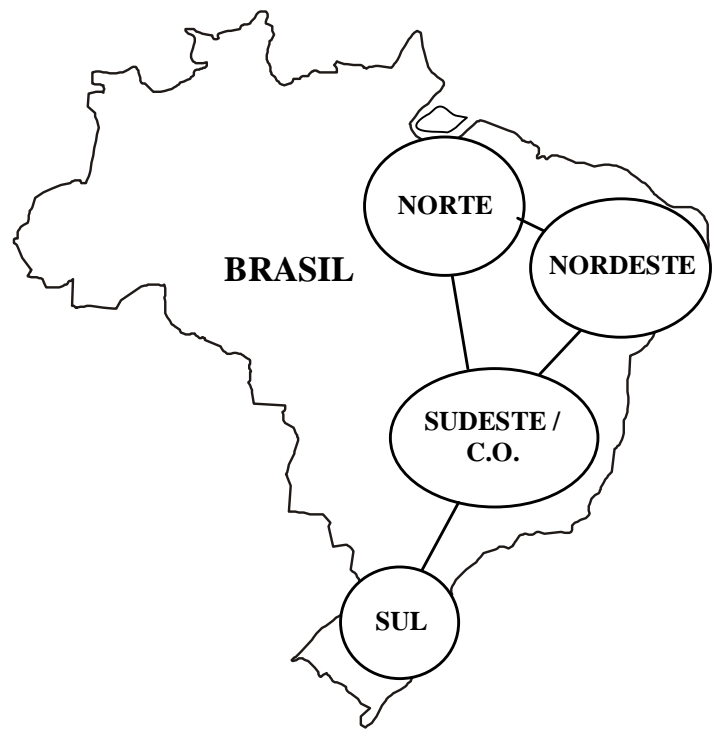

Figura 1: Sistema interligado nacional brasileiro

áreas as grandes regiões do sistema: Norte, Nordeste, Sul e Sudeste/Centro-Oeste. Estas regiões estão interligadas entre si como mostra a figura 1. A geração de Itaipu foi considerada pertencente a região Sudeste/ Centro-Oeste.

A tabela 1 apresenta a participação de cada região do sistema em termos de carga e geração. Em termos percentuais a região Norte é responsável por 5,16\% de toda demanda de potência ativa do sistema, Nordeste $14,48 \%$, Sul $17,73 \%$ e a região Sudeste/Centro-Oeste atende $62,62 \%$ de toda carga do sistema.

A tabela 2 apresenta o intercâmbio de potência ativa entre estas regiões para este caso de estudo. Pode-se observar que, para este cenário, as regiões Norte e Sudeste/Centro-Oeste são exportadoras de energia para as regiões Nordeste e Sul. Os valores positivos e negativos nas tabelas indicam exportações e importações respectivamente relativas à região, indicada na linha da tabela.

Tabela 1: Geração e demanda de potência das regiões

\begin{tabular}{|c|c|c|c|c|}
\hline \multirow{2}{*}{ Região } & \multicolumn{2}{|c|}{ Carga } & \multicolumn{2}{c|}{ Geração } \\
\cline { 2 - 5 } & MW & Mvar & MW & Mvar \\
\hline Norte & $2.987,5$ & 661,6 & $5.994,0$ & $-301,7$ \\
\hline Nordeste & $8.381,3$ & $2.225,4$ & $8.047,1$ & $-624,9$ \\
\hline Sul & $10.260,3$ & $2.676,8$ & $9.075,6$ & 333,9 \\
\hline $\begin{array}{c}\text { Sudeste/ } \\
\text { C.O. }\end{array}$ & $36.237,1$ & $9.988,9$ & $37.899,9$ & $1.186,5$ \\
\hline Total & $57.866,1$ & $15.552,7$ & $61.016,6$ & 593,8 \\
\hline
\end{tabular}

\begin{tabular}{|c|c|c|c|c|}
\hline & Norte & Nord. & Sul & $\begin{array}{c}\text { Sudeste/ } \\
\text { C.O. }\end{array}$ \\
\hline Norte & - & 804,5 & - & $1.980,5$ \\
\hline Nordeste & $-804,5$ & - & - & 104,0 \\
\hline Sul & - & - & - & $-1.642,2$ \\
\hline $\begin{array}{c}\text { Sudeste/ } \\
\text { C.O. }\end{array}$ & $-1.980,5$ & $-104,0$ & $1.642,2$ & - \\
\hline
\end{tabular}

\subsection{Avaliação da MET}

A margem de estabilidade de tensão do SIN foi avaliada considerando, além dos limites de geração de potência reativa dos geradores, os seguintes controles:

- Controle de tensão por variação automática de tap dos transformadores dentro dos limites físicos destes equipamentos;

- Controle de tensão por injeção remota de potência reativa dentro dos limites mínimos e máximos.

Considerando estas opções de controle ativas para se obter resultados mais realistas e utilizando o método da continuação como mencionado na seção 2.1 deste artigo, a margem de estabilidade de tensão do sistema foi avaliada em $0,62 \%$.

Para uma análise mais detalhada, faz-se necessário avaliar a margem de estabilidade de tensão de cada região do sistema. Esta margem de estabilidade indica o quanto o carregamento da região de estudo poderá aumentar, sem que seja necessário auxílio de geração das regiões externas à área de estudo para atender o novo carregamento do sistema. Como apenas a geração da área em estudo irá suprir o acréscimo na demanda desta área, o intercâmbio entre as regiões permanece constante, sendo o mesmo do apresentado na tabela 2.

A figura 2 apresenta estes resultados. Nota-se claramente que a região Sudeste/Centro-Oeste é a que possui menor margem de estabilidade de tensão, com valor muito baixo de apenas $0,68 \%$. Pode-se observar ainda que a margem total do sistema $(0,62 \%)$ é limitada pela região de menor margem de estabilidade, que no caso é a região Sudeste/Centro-Oeste.

A figura 3 apresenta as perdas de potência ativa para cada região do sistema. Nota-se que a região Sudeste/Centro-Oeste apresenta maior nível de perdas de potência ativa, tanto em valores absolutos como em percentuais. Esta região detém mais da metade de toda demanda do sistema, estando mais estressada do que as outras regiões. Como apresentado em 


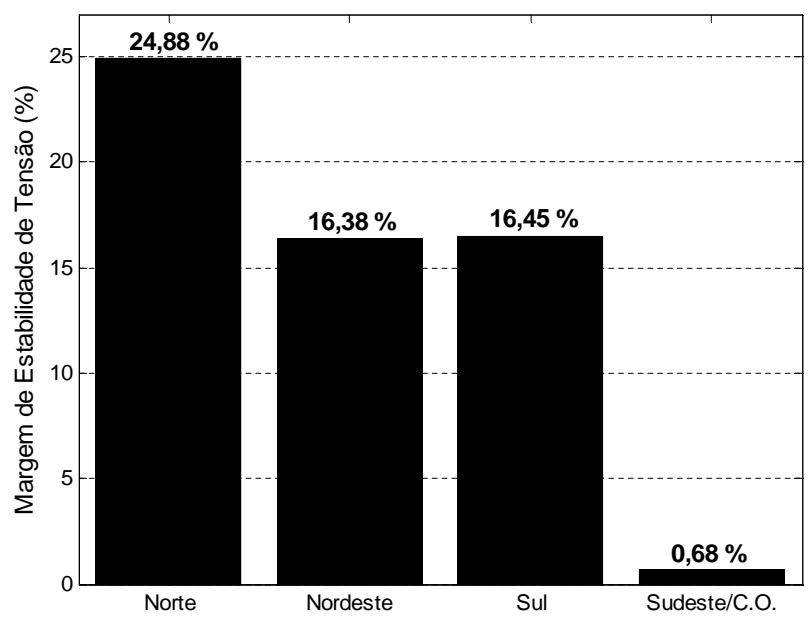

Figura 2: MET para cada região do SIN

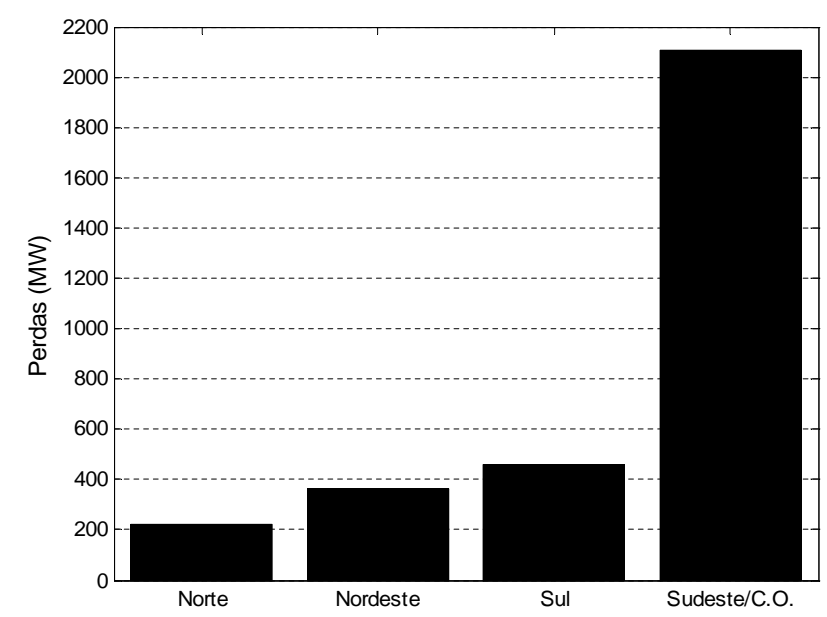

Figura 3: Perdas de potência ativa para cada região do SIN

(Affonso and Da Silva, 2004), existe um impacto das perdas na MET. A minimização das perdas de potência ativa conduz a maximização da margem de estabilidade de tensão e vice-versa. A figura 4 apresenta a curva PV para a região Sudeste/Centro-Oeste para a barra Ouro Preto 345kV.

Os resultados mostram que a região Sudeste/Centro-Oeste apresenta menor margem de estabilidade de tensão, com valor muito baixo, de apenas $0,68 \%$, e que esta região limita o valor da margem de estabilidade de todo o sistema. Sendo assim, faz-se necessário realizar um estudo detalhado desta região identificando suas áreas críticas bem como possíveis ações para melhoria da estabilidade do sistema, tanto em condições normais de operação como quando sujeito a contingências.

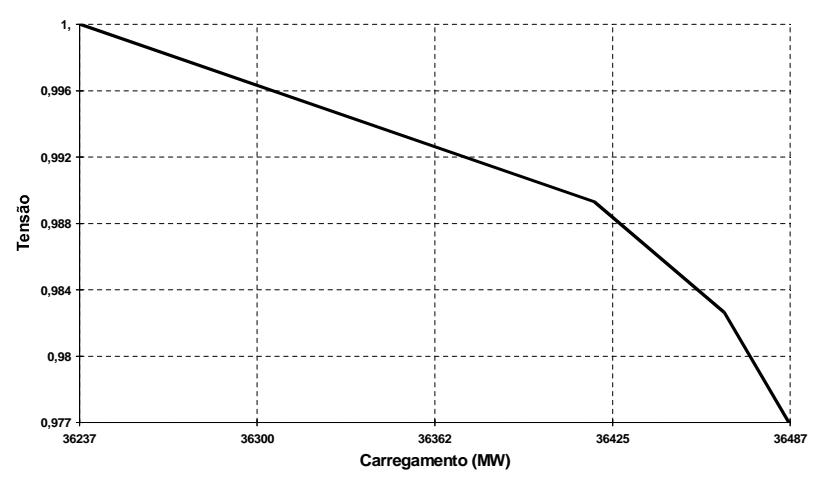

Figura 4: Curva PV para a região Sudeste/ Centro-Oeste.

\section{AVALIAÇÃO DA ESTABILIDADE DE TENSÃO DA REDE DE TRANSMISSÃO DO ESTADO DE SÃO PAULO}

Devido a indisponibilidade de um sistema representativo da região Sudeste/Centro-Oeste, foram realizados estudos utilizando a rede de transmissão do estado de São Paulo com dados de operação disponibilizados pela CTEEP (Companhia de Transmissão de Energia Elétrica Paulista).

\subsection{Sistema de transmissão de São Paulo}

O mercado de energia elétrica do Estado de São Paulo é atendido em grande parte pelas usinas dos rios Paraná, Paranapanema e Tietê com potência instalada da ordem de 10.700 MW, usinas do rio Paraíba do Sul com 170 MW instalados, usinas do rio Pardo com 220 MW instalados, complexo de Henry Borden com cerca de 900 MW instalados, usinas térmicas da área com cerca de $700 \mathrm{MW}$ instalados, usina de Itaipu com 12.600 MW instalados, bem como por outras usinas dos rios Grande e Paranaíba. O sistema de transmissão da rede básica que atende o estado de São Paulo é constituído principalmente por:

- Três circuitos em $765 \mathrm{kV}$ (UHE Itaipu - 60Hz)

- Elo CC em 600kV (UHE Itaipu - 50Hz)

- Circuitos em 500kV, 440kV, 345kV e 230kV

\subsection{Avaliação da MET}

Utilizando os dados da rede para os dias 18 e 19 de fevereiro de 2004, obteve-se a margem de estabilidade de tensão para diversos intervalos, formando um dia completo. A dificuldade em obter os dados da rede do Estado de São Paulo contribuiu para que um dia de estudo fosse formado por períodos dos dias 18 e 19 de fevereiro de 2004. Estes dias antecederam 


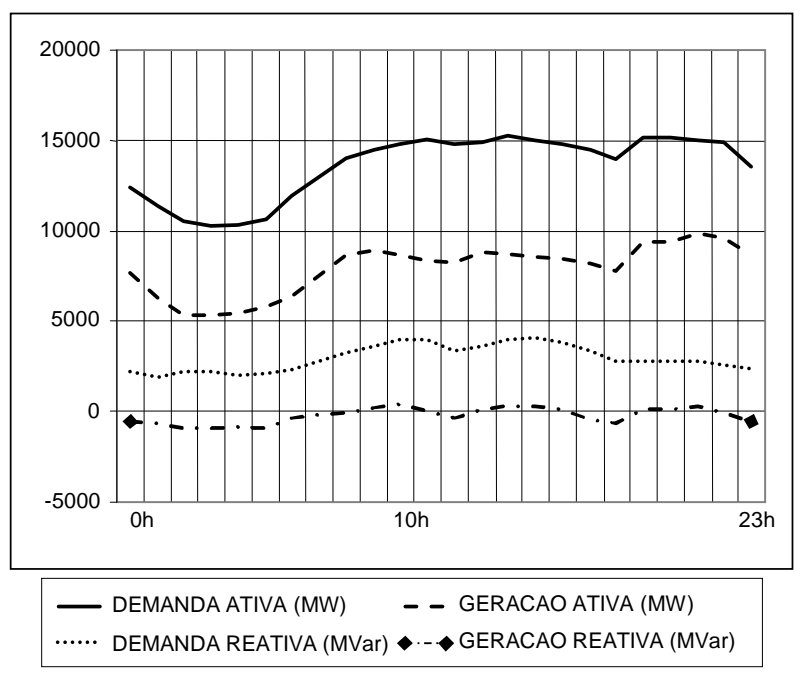

Figura 5: Demanda e geração para intervalos de um dia.

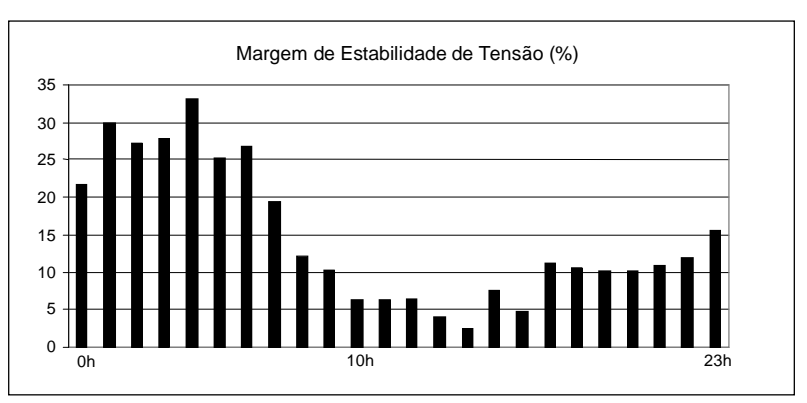

Figura 6: Comportamento da MET para intervalos de um dia.

o feriado de carnaval (24 de fevereiro), com possíveis alterações no comportamento da demanda e resultados diferentes se comparados a um dia típico de operação.

A figura 5 apresenta a variação de demanda e geração na área de São Paulo durante o período de um dia e a figura 6 ilustra o comportamento da MET avaliada para cada horário. Observa-se que as piores MET e as maiores demandas de potência ativa e reativa ocorrem fora do período de ponta (entre as 18 e 20 horas) e sim próximo as 14 horas do dia 19 de fevereiro, o que pode ser explicado por se tratar de um dia atípico.

Para a rede elétrica do Estado de São Paulo os valores da MET para alguns horários estão abaixo das recomendações do WECC e ONS, que sugerem uma MET mínima de 5\% e $6 \%$, respectivamente, mesmo considerando a contingência simples mais severa (N-1). Os valores de MET encontrados para o período de carga leve são satisfatórios devido ao baixo carregamento deste período, que fazem com que as linhas de transmissão operem com característica capacitiva, elevando o perfil de tensão da rede. Nestas situações, ações de redu- ção da tensão nas barras de controle são necessárias, como por exemplo, redução da tensão nos terminais dos geradores, inserção de reatores e até mesmo, o desligamento de algumas linhas de transmissão. Na situação de carga média a demanda de potência ativa e reativa aumenta reduzindo a MET.

\subsection{Análise de Contingências}

Muitos incidentes de estabilidade de tensão resultam de grandes distúrbios tais como perda da transmissão ou equipamentos de geração, usualmente combinados com elevado carregamento. Visto que situações de contingências podem ter impacto negativo na estabilidade, levando o sistema eventualmente ao colapso, esta análise é de grande importância para garantir a operação segura da rede.

Para medir o grau de segurança pós-contingência (N-1) da operação do sistema elétrico de São Paulo em relação à estabilidade de tensão, a MET foi calculada via curva PV para diversas contingências, para os diversos horários de um dia. Foram analisadas somente contingências simples e relacionadas à saída de ramos pertencentes à área São Paulo e aqueles que ao menos uma de suas barras terminais pertença à área interna (ramos de intercâmbio).

A figura 7 apresenta o máximo carregamento do sistema obtido para cada uma das 900 contingências simuladas às 12 horas do dia 19 de fevereiro de 2004. Na figura também são apresentados, incicados por linhas pontilhadas, o carregamento inicial e o máximo carregamento do caso base. Considera-se como caso base a operação do sistema no dia 19 de fevereiro de 2004 às 14 horas, sem a ocorrência de nenhuma contingência. A MET é então dada pela diferença entre o máximo carregamento e o carregamento inicial, cujo valor é de $6,35 \%$. Observa-se que apenas um número bastante reduzido de contingências causaria uma redução significativa no valor da margem de estabilidade.

A avaliação apenas do valor da margem de estabilidade de tensão pós-contingência não fornece informação suficiente para mensurar a severidade da contingência em questão. Isto porque, independente do impacto no valor da margem de estabilidade, as contingências podem ser de abrangência local, de área ou sistêmica. Em contingências com abrangência local apenas algumas barras na vizinhança da contingência são afetadas. Em contingências com abrangência de área algumas barras pertencentes a área em que ocorreu a contingência são afetadas. Em contingências com abrangência sistêmica diversas barras de todo o sistema são afetadas, oferecendo sim neste caso risco de um colapso de tensão no sistema. Sendo assim, uma contingência pode provocar forte impacto na margem, mas ao mesmo tempo ter abrangência extremamente reduzida a algumas barras da rede, não configurando, portanto, uma contingência severa. 


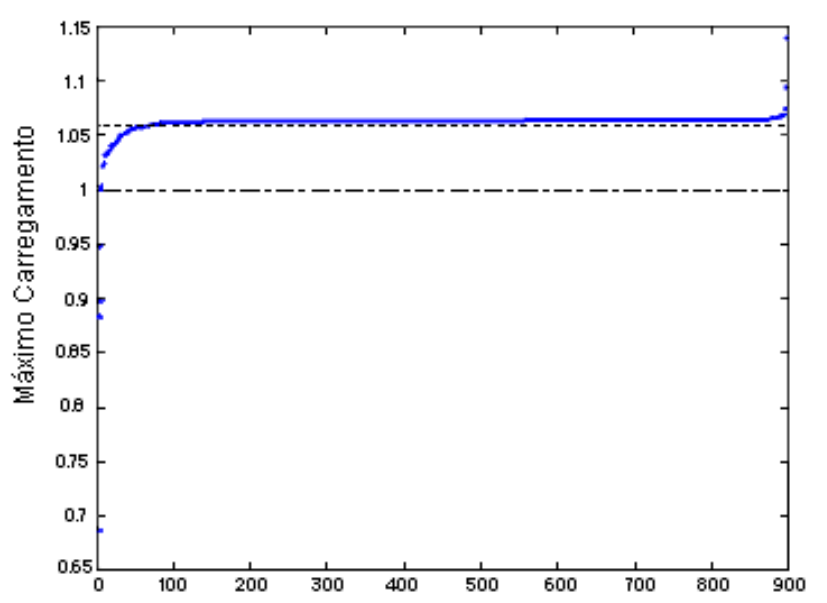

Figura 7: Máximo carregamento para diversas contingências.

Utilizando a metodologia de análise modal pode-se verificar a abrangência das contingências capturadas através do método de curva PV, possibilitando mensurar o impacto como local, de área ou sistêmico. Cada contingência é analisada através da análise modal. O espectro modal permite a visualização gráfica dos fatores de participação normalizados, indicando as barras que mais participam do modo crítico, ou seja, os pontos preferenciais da rede para aplicação das ações de controle ou de reforço. Estes resultados são extremamente importantes para a interpretação adequada do valor obtido para a margem de estabilidade de tensão de um dado ponto de operação.

Os resultados mostram que grande parte das contingências com relevante impacto na MET do sistema apresenta apenas abrangência localizada em torno das primeiras vizinhanças das barras associadas à contingência. As contingências com abrangência sistêmica não produzem impacto significativo na margem de estabilidade por fazerem parte de uma malha redundante que oferece caminhos alternativos ao fluxo de potência sem depreciar de forma significativa às reservas de potência reativa do sistema. Para ilustrar estas conclusões, a análise de duas contingências será apresentada.

\subsubsection{Perda da linha entre Aparecida e Taubaté 230kV}

Como exemplo de uma contingência com grande impacto na MET porém de abrangência local, apresenta-se a contingência entre Aparecida e Taubaté $230 \mathrm{kV}$ simulada às $13 \mathrm{~h}$ do dia $19 / 02$. Esta contingência reduz significativamente a margem de estabilidade de 3,91\% para $-1,09 \%$. A figura 8 e 9 apresentam os fatores de participação ativo e reativo respectivamente para todas as barras de carga da rede após a ocorrência desta contingência. Analisando apenas o valor da MET póscontingência, teria-se esta contingência como severa. Nota-

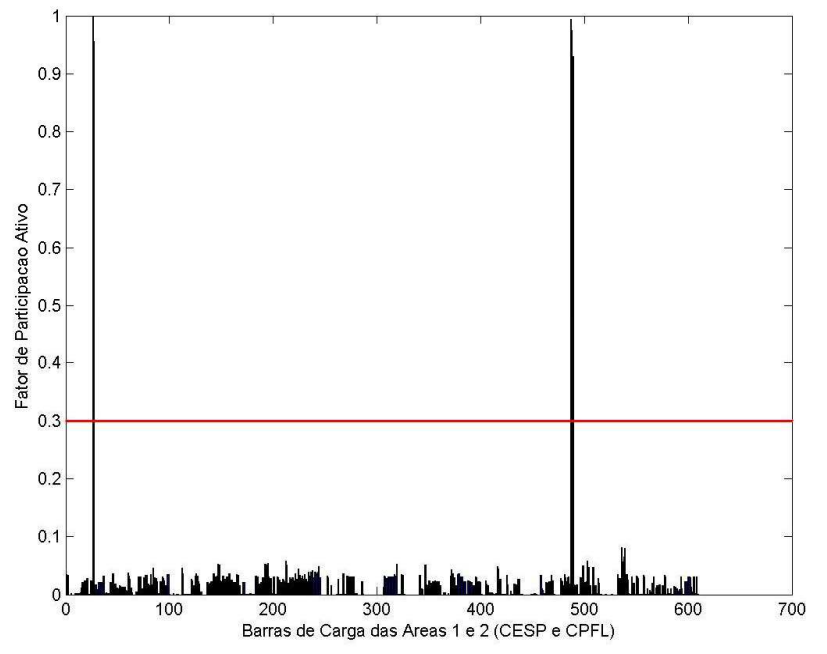

Figura 8: FPA (contingência LT Aparecida/Taubaté 230kV)

se, no entanto, que apenas algumas barras apresentam fator de participação pós-contingência elevado, sendo portanto esta contingência de abrangência localizada. Isto significa que medidas corretivas de corte de carga ou injeção de potência reativa apenas em algumas barras da rede aumentariam a MET e o sistema continuaria operando de forma segura. As barras preferenciais para aplicação das ações de controle ou de reforço são aquelas que apresentam os maiores FPA e FPR, responsáveis por maiores participações no modo crítico.

Para ilustrar o impacto localizado desta contingência, realizou-se um corte de carga de forma escalonada e com fator de potência constante para as barras críticas de $88 \mathrm{kV}$ de alto FPA. Percebe-se, através da figura 10 que há uma grande recuperação da margem do sistema quando os cortes de carga são realizados nas barras críticas obtidas através da análise modal pós-contingência. Ou seja, a redução na MET devido à contingência é elevada, no entanto sua recuperação é simples e direta através de ações corretivas na vizinhança da contingência, nas barras indicadas pelos fatores de participação.

A figura 11 apresenta o comportamento da MET com a inserção de potência reativa na barra de Taubaté, realizada apenas para fins ilustrativos para verificar como o sistema irá responder no que se refere a MET com a inserção de potência reativa nesta barra. Esta barra possui maior FPR, sendo portanto a melhor opção para aplicação de reforço a fim de melhorar a margem de estabilidade de tensão da rede. Verifica-se a melhoria expressiva da margem de estabilidade através da inserção de blocos de 50Mvar, representando o chaveamento de bancos de capacitores shunt. 


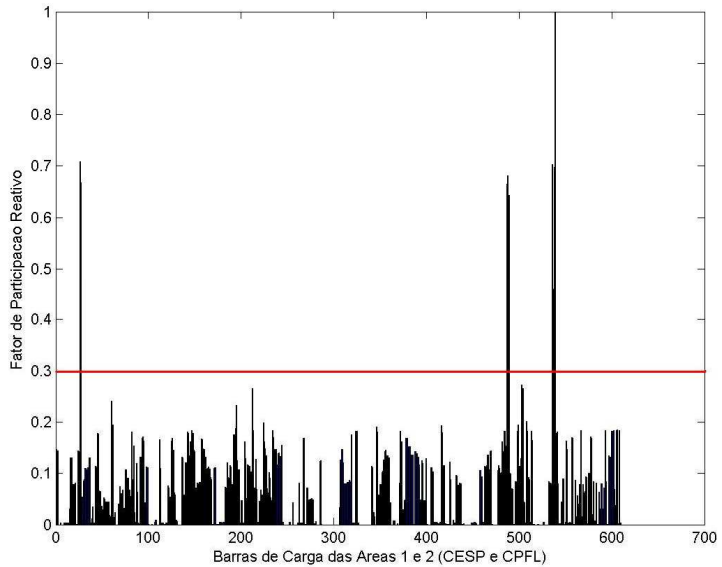

Figura 9: FPA (contingência LT Aparecida/Taubaté 230kV)

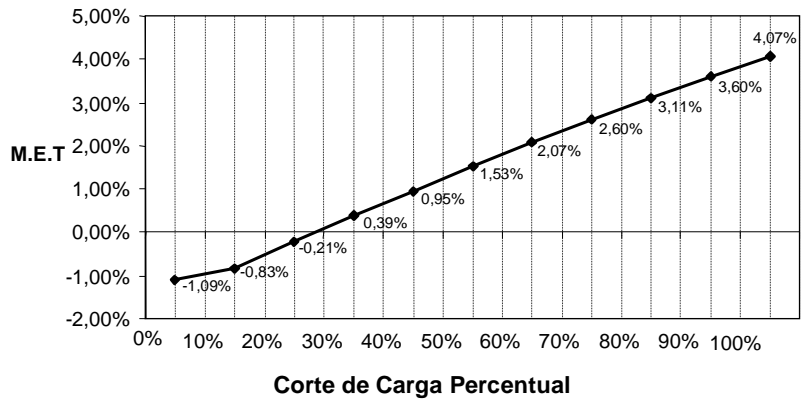

Figura 10: Aumento da MET para corte de carga incremental.

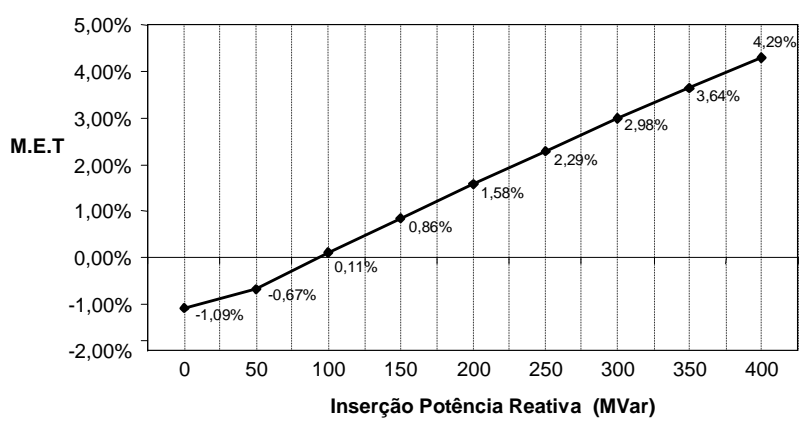

Figura 11: Aumento da MET para inserção de potência reativa

\subsubsection{Perda da linha entre Araraquara e Santa Bár- bara $440 \mathrm{kV}$}

Para exemplificar uma contingência de abrangência sistêmica, são apresentados os resultados obtidos com a simulação da contingência da linha de transmissão entre Araraquara e Santa Bárbara $440 \mathrm{kV}$ às $14 \mathrm{~h}$ do dia 19 de fevereiro. A MET pré-contingência é de $2,45 \%$, sendo reduzida para

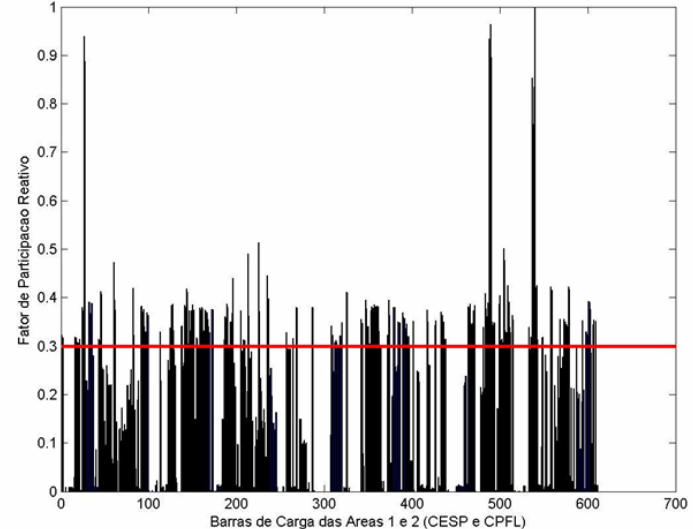

Figura 12: FPR (contingência LT Araraquara/Santa Bárbara $440 \mathrm{kV})$

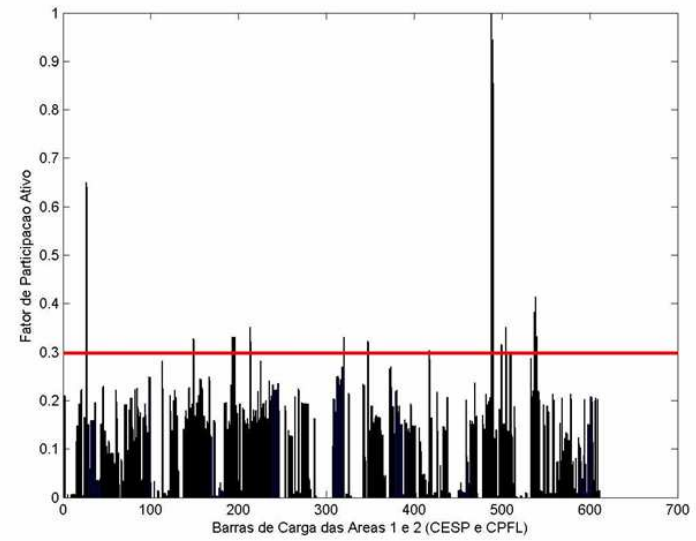

Figura 13: FPA (contingência LT Araraquara/Santa Bárbara $440 \mathrm{kV})$

$0,6 \%$ com a ocorrência desta contingência. As figuras 12 e 13 ilustram os fatores de participação reativo e ativo respectivamente, após a perda da linha de transmissão entre Araraquara e Santa Bárbara 440kV. Nota-se que o espectro de participação reativo é bastante abrangente, visto que a maior parte das barras do sistema apresenta FPR maior do que o FPR médio. Já o espectro de participação ativo é mais localizado, pois o número de barras com FPA maior do que o FPA médio é bem menor.

Analisando apenas o valor da margem pós-contingência, a perda da linha Aparecida/Taubaté $230 \mathrm{kV}$, com margem póscontingência de $-1,09 \%$, seria bem mais severa do que a perda da linha Araraquara/Santa Bárbara 440kV, com margem pós-contingência de $2,45 \%$. No entanto, a ocorrência da contingência Aparecida/Taubaté $230 \mathrm{kV}$ exige ações de refor- 
ços localizadas na rede para a recuperação da estabilidade do sistema, sendo de simples solução. Já a ocorrência da contingência Araraquara/Santa Bárbara $440 \mathrm{kV}$ requer ações de reforços distribuídas em diversos pontos da rede para melhoria da estabilidade de tensão de todo o sistema, sendo esta, portanto, uma contingência mais severa.

\section{CONCLUSÃO}

Este artigo apresenta resultados de um diagnóstico sobre o nível de segurança com que o sistema interligado brasileiro opera em relação à estabilidade de tensão, utilizando dados reais do planejamento e da operação. Os resultados mostram que o sistema opera com margem de estabilidade baixa, de apenas $0,62 \%$ para o caso base (sem ocorrência de contingências). A margem de estabilidade de todo sistema é limitada pela região Sudeste/Centro-Oeste que apresenta maiores perdas de potência ativa e menor MET.

Um estudo mais detalhado foi apresentado para a rede de transmissão do estado de São Paulo, avaliando o comportamento da margem de estabilidade ao longo de um dia e após a ocorrência de diversas contingências. Com os resultados pode-se observar que a maior parte das contingências com impacto relevante na margem de estabilidade de tensão apresenta apenas abrangência localizada, atingindo barras nas vizinhanças à contingência. Por outro lado, as contingências com abrangência sistêmica não produzem impacto significativo na MET por fazerem parte de uma malha redundante que oferece caminhos alternativos ao fluxo de potência sem depreciar de forma significativa as reservas de potência reativa do sistema. Deste modo, quanto ao risco de colapso de tensão sistêmico pode-se concluir que o sistema do estado de São Paulo está bem resguardado, podendo perder elementos simples das principais malhas de alta tensão sem impactar significativamente na margem de estabilidade.

Em termos do problema de estabilidade de tensão considerando o critério (N-1), o sistema elétrico de São Paulo pode se tornar mais seguro ainda através da simples aplicação de reforços localizados em alguns pontos do sistema, de forma a evitar desligamentos localizados em torno destas áreas. Os locais mais adequados para a aplicação de reforços (injeção de potência reativa e corte de carga) foram identificados através dos fatores de participação, os quais mostraram-se adequados.

Os resultados desta pesquisa indicam a necessidade de uma reinterpretação conceitual da margem de estabilidade do sistema, pois do ponto de vista prático, o valor da margem para casos (N-0) e (N-1) é pouco significativo se não vier acompanhada de uma análise da área de impacto da contingência sobre o sistema. Uma contingência pode provocar forte impacto na margem mas ao mesmo tempo ter abrangência extremamente reduzida a algumas barras da rede, não configurando, portanto, uma contingência severa e não oferece risco de um colapso de tensão sistêmico. Isto porque na prática, não haveriam desligamentos em cascata e tampouco um colapso de tensão, pois estas poucas barras seriam desligadas por sub-tensão e o sistema continuaria operando com segurança. Isto explica a não ocorrência de problemas de estabilidade de tensão na rede de São Paulo no período de estudo analisado, mesmo o sistema apresentando margem de estabilidade muito baixa.

\section{REFERÊNCIAS}

Affonso C.M., Da Silva L.C.P., Lima F.G.M. and Soares S. (2004). MW and MVar Management on Supply and Demand Side for Meeting Voltage Stability Margin Criteria, IEEE Transactions on Power Systems, Vol. 19, No. 3 , pp. 1538-1545.

Affonso C. M. and Da Silva L.C.P. (2004). Voltage Stability Improvement by Optimizing Reactive Power Reserves, Proceedings of the Fourth IASTED International Conference - Power and Energy Systems, Rhodes, Greece, pp. 394-399.

Amjady N. (2004). A Framework of Reliability Assessment With Consideration Effect of Transient and Voltage Stabilities, IEEE Transactions on Power Systems, Vol. 19, No. 2, pp. 1005-1014.

Bao L., Huang Z. and Xu W. (2003). Online Voltage Stability Monitoring Using Var Reserves. IEEE Transactions on Power Systems, Vol. 18, No. 4, pp. 1461 - 1469.

CEPEL (2004). Programa de Análise de Redes / ANAREDE - Manual do Usuário Versão 08-8/2004. Rio de Janeiro, RJ, Brasil.

Corsi S. and Sabelli, C. (2004). General Blackout in Italy Sunday September 28, 2003, h. 03:28:00, Proceedings of IEEE Power Engineering Society General Meeting, Vol. 2, pp.1691-1702.

Da Silva L.C.P., Wang Y., Da Costa V.F. and Xu W. (2002). Assessment of generator impact on system power transfer capability using modal participation factors, Proc. IEE Gener. Transm. Distrib., Vol. 149, 2002.

Força Tarefa (1999). Critérios e Metodologias Estabelecidos no âmbito da Força - Tarefa Colapso de Tensão do GTAD/SCEL/GCOI para Estudos de Estabilidade de Tensão nos Sistemas Interligados Norte/Nordeste, Sul/Sudeste e Norte/Sul Brasileiros, Anais do XV SNPTEE, Foz do Iguaçu, PR, Brasil.

IEEE/CIGRE Joint Task Force on Stability Terms and Definitions (2004). Definition and Classification of Power 
System Stability, IEEE Transactions on Power Systems, Vol. 19, No. 2, pp. 1387-1401.

Kabouris, J., Vournas, C.D., Efstathiou, S., Manos, G.A. and Contaxis, G.C. (2000). Voltage Security Considerations in an Open Power Market, Proceedings of IEEE International Conference on Electric Utility Deregulation and Restructuring and Power Technologies, pp. 278283.

Kamalasadan S., Srivastava A. K., Thukaram D. (2006). Novel Algorithm for Online Voltage Stability Assessment Based on Feed Forward Neural Network. Proc. IEEE

Power Engineering Society General Meeting, pp. 1 - 7.

Kundur, P. (1994). Power System Stability and Control. Ed. New York: McGraw-Hill.

ONS (2002) Procedimentos de Rede Módulo 21: Estudos Especiais. Submódulo 21.5: Estudos de Estabilidade de Tensão. http://www.ons.org.br

Taylor, C.W. (1994). Power System Voltage Stability, New York: McGraw-Hill Inc.

U.S.-Canada Power System Outage Task Force (Apr., 2004). Final Report on the August 14, 2003 Blackout in the United States and Canada: Causes and Recommendations. http://www.iwar.org.uk/cip/resources/blackout-03/

WECC Reactive Power Reserve Work Group (1998). Final Report: Voltage Stability Criteria, Undervoltage Load Shedding Strategy, and Reactive Power Reserve Monitoring Methodology. http://www.wecc.biz 